\title{
Fluctuations of Macrovariables in Nonlinear Systems: Langevin Equation Approach
}

\author{
Ziya A. Akcasu ${ }^{1}$
}

Received June 23, 1976

Fluctuations in nonlinear Markovian systems are studied by the Langevin equation method using system-size expansion. Langevin equations with different random sources are constructed for the description of fluctuations to varying degrees of accuracy in inverse powers of the system size $\epsilon$. Evolution equations for the deterministic path, deviation of the mean from the deterministic path, and the variance are obtained in a nonstationary state in the lowest order of $\epsilon$. The power spectral density for fluctuations about a stable equilibrium is calculated correct to order $\epsilon^{2}$ and is compared to the exact expression for the Alkemade diode. The relaxation frequency for the decay of correlations in a critical equilibrium and the scaling law for the anomalous fluctuations are determined and compared to those obtained by Kubo et al.

KEY WORDS : Fluctuations far from equilibrium ; Markovian processes ; Langevin equation ; Alkemade diode ; system-size expansion ; relaxation of fluctuations in critical states; nonlinear reactor noise.

\section{INTRODUCTION}

In this paper we study the fluctuations of macrovariables in nonlinear macroscopic systems with the Langevin equation method. A macrovariable is defined as an extensive quantity characterizing, along with other similar quantities, a macrostate of the macroscopic system consisting of a large number of interacting or noninteracting elementary subsystems. The number of neutrons in a nuclear reactor, the total magnetization in a ferromagnet, and the population of a given kind in a microbial community are examples

Supported by National Science Foundation.

\footnotetext{
${ }^{1}$ Department of Nuclear Engineering, The University of Michigan, Ann Arbor, Michigan.
} 
of macrovariables in physical and nonphysical systems. The characteristic feature of such variables is that they are a superposition of a large number of microscopic quantities, and they display fluctuations about their statistical average when considered as a function of time. These fluctuations are regarded as a random process. The macroscopic system may be in thermal equilibrium, or just in a steady state, or even in a nonstationary state. In any case, one is interested in determining the joint probability distribution function describing the statistics of the fluctuations of macrovariables in the system as a function of time. In some instances one is satisfied only with the calculation of twotime correlations of pairs of dynamical variables when the complete statistical problem is insoluble.

In the macroscopic theory of fluctuations one usually assumes that the fluctuations of the macrovariables constitute a Markovian random process, and starts with the master equation describing such processes in terms of transition probabilities per unit time. Van Kampen ${ }^{(1)}$ showed, using the Kramers-Moyal expansion of the master equation in powers of $\epsilon=1 / \Omega$, where $\Omega$ is the size of the system, which is assumed to be uniform, that the master equation reduces in the lowest order in $\epsilon$ to a linear Fokker-Planck equation with time-dependent coefficients, and the probability distribution of the macrovariables about the most probable path is Gaussian. More specifically, he concluded that it is inconsistent to use the nonlinear Fokker-Planck equation in nonlinear systems without including at the same time higher derivatives of the distribution function than the second. More recently Kubo et al. ${ }^{(2)}$ obtained time evolution equations for the most probable path (or deterministic motion), variance, and deviation of the mean from the deterministic path by first proving conservation of the extensive property of the distribution function $P(X, t)$ of a macrovariable in time and then using an expansion of $\ln P(X, t)$ again in powers of $\epsilon$. They illustrated the application of the evolution equations to the Weiss-Ising model and discussed some aspects of anomalous fluctuations about a marginal and critical equilibrium. Their approach also is applicable to uniform systems only. Mori ${ }^{(3)}$ proposed a scale transformation of a nonequilibrium macroscopic state to obtain a kinetic equation for the evolution and fluctuations of the macrovariables in nonuniform systems. Haken ${ }^{(4)}$ reviewed techniques for investigating cooperative phenomena in physical systems far from equilibrium and in nonphysical systems. Recently, Keizer, ${ }^{(4)}$ in a series of papers, has developed a purely phenomenological theory of fluctuations in nonlinear macroscopic systems in terms of three postulates.

The generally accepted idea of the Langevin equation method which we use in this paper is that fluctuations of a macrovariable can be taken into account by simply adding a random source (Langevin assumption) to the macroscopic rate equation, which is usually known phenomenologically. Although the physical meaning of this source is often obscure, one assumes 
it to be a white noise process with zero mean obeying the causality condition. In stationary linear systems, characterized by a linear macroscopic rate equation, the application of Langevin technique is straightforward. However, in nonlinear systems the noise source is generally nonstationary and its spectral density is dependent on the state of the system, so that the application of the Langevin technique to such systems ceases to be straightforward. van Kampen ${ }^{(5)}$ has elucidated the difficulties in the application of Langevin method to nonlinear macroscopic systems from the point of view of systemsize expansion. Since many recent treatments of nonlinear processes use the Langevin equation method, we make an attempt in this paper to investigate its foundation and validity for studying fluctuations in nonlinear systems. We restrict ourselves to Markov processes and develop, by means of system-size expansion, the stochastic equations describing the fluctuations to varying degrees of accuracy in powers of $\epsilon$. These equations contain different random sources, and constitute the Langevin equations for a nonlinear system. In the description to lowest order in $\epsilon$ the theory reproduces evolution equations for the deterministic path, variance, deviation of the mean from the deterministic path, the correlation function, scaling rules for anomalous fluctuations, and the relaxation frequency of their correlation function in a critical equilibrium, as obtained by Kubo et al. ${ }^{(2)}$ The next higher order description of fluctuations enables one to calculate the power spectral density in stable equilibrium up to order $\epsilon^{2}$. When applied to Alkemade's diode ${ }^{(6)}$ as a nonlinear model system, the latter reproduces the power spectral density obtained by van Kampen ${ }^{(7,8)}$ by expanding the exact spectrum in powers of $\epsilon$ and retaining the first two terms. On the basis of these results we conclude that the Langevin equation method is sound and indeed powerful to study fluctuations in nonlinear systems.

In Section 2, we present the moment equations of a Markov process, which are later used to develop the stochastic equations. In Section 3 we discuss the foundation of the Langevin equation method as an alternative approach to the study of fluctuations in nonlinear systems, and obtain the properties of the Langevin source appearing in the nonlinear macroscopic equation. In this section in particular we follow Lax ${ }^{(9)}$ very closely. In Section 4 we use van Kampen' ${ }^{(1)}$ system-size expansion to obtain the evolution and stochastic equations in increasing orders of $\epsilon$. In Section 5 we study fluctuations and the evolution of the mean using the lowest order Langevin description. Section 6 is devoted to the study of fluctuations about equilibrium using the next higher order Langevin description. The application of the general results to nonlinear model systems, such as Alkemade's diode, the Weiss-Ising model, and nuclear reactors, is presented in Section 7. Finally, in Section 8 we discuss scaling of fluctuations and time in the case of a critical equilibrium. The conclusions and discussions appear in Section 9 . 


\section{MOMENT EQUATIONS}

Let $X(t)$ be a macrovariable in a uniform system of size $\Omega$, and assume that its fluctuations are described by a Markov process with the master equation

$$
\frac{\partial P(X, t)}{\partial t}=\int d r[W(X-r, r, t) P(X-r, t)-W(X, r, t) P(X, t)]
$$

where $P(X, t)$ is the probability density at time $t$ and $W(X, r, t)$ is the transition probability per unit time at $t$ from $X$ to $X+r$ with a jump $r$. We assume that $W$ obeys

$$
W(X, r, t)=\Omega \omega(x, r, t)
$$

where $x=X / \Omega$ is the intensive variable and $\omega(x, r, t)$ does not depend on $\Omega$ explicitly. The master equation (1) is written in terms of $x$ and $\omega$ as

$$
\epsilon \frac{\partial P(x, t)}{\partial t}=\int d r[\omega(x-\epsilon r, r, t) P(x-\epsilon r, t)-\omega(x, r, t) P(x, t)]
$$

where $\epsilon=\Omega^{-1}$ and $P(x, t)=\Omega P(X, t)$. The moments of the transition probability are defined by

$$
c_{n}(x, t)=\int d r r^{n} \omega(x, r, t)
$$

Clearly the $c_{n}(x, t)$ also do not depend on $\epsilon$ explicitly. In a time-invariant system the transition probability $\omega$ and, hence, $c_{n}$ are independent of time.

The moments of $x(t)$ are obtained from (3) as

$$
\begin{aligned}
d\langle x(t)\rangle / d t= & \left\langle c_{1}[x(t), t]\right\rangle \\
d\left\langle x^{2}(t)\right\rangle / d t= & 2\left\langle c_{1}[x(t), t] x(t)\right\rangle+\epsilon\left\langle c_{2}[x(t), t]\right\rangle \\
d\left\langle x^{3}(t)\right\rangle / d t= & 3\left\langle c_{1}[x(t), t] x^{2}(t)\right\rangle+3 \epsilon\left\langle c_{2}[x(t), t] x(t)\right\rangle \\
& +\epsilon^{2}\left\langle c_{3}[x(t), t]\right\rangle
\end{aligned}
$$

where we define

$$
\left\langle c_{j}[x(t), t] x^{n}(t)\right\rangle=\int d x c_{j}(x, t) x^{n} P(x, t)
$$

The transition probability $P\left(x, t \mid x^{\prime}, t^{\prime}\right)\left(t>t^{\prime}\right)$ of the processes satisfies (3), as a function of $t$, with the initial condition $P\left(x, t \mid x^{\prime}, t\right)=\delta\left(x-x^{\prime}\right)$. The Markov property implies

$$
\begin{aligned}
& P_{n}\left(x_{1}, t_{1} ; x_{2}, t_{2} ; \ldots ; x_{n}, t_{n}\right) \\
& \quad=P\left(x_{1}, t_{1}\right) P\left(x_{2}, t_{2} \mid x_{1}, t_{1}\right) \ldots P\left(x_{n}, t_{n} \mid x_{n-1}, t_{n-1}\right)
\end{aligned}
$$


where $t_{n}>t_{n-1}>\cdots>t_{1}$ and $P_{n}$ is the joint probability density at $t_{1}, t_{2}, \ldots, t_{n}$. Using $P_{2}$, one defines the correlation function of the process for $t>t^{\prime}$ as

$$
\left\langle x(t) x\left(t^{\prime}\right)\right\rangle=\int d x^{\prime} x^{\prime} P\left(x^{\prime}, t^{\prime}\right) \int d x x P\left(x, t \mid x^{\prime}, t^{\prime}\right)
$$

Differentiating (9) with respect to $t$ and using the master equation, one obtains

$$
\partial\left\langle x(t) x\left(t^{\prime}\right)\right\rangle / \partial t=\left\langle c_{1}[x(t), t] x\left(t^{\prime}\right)\right\rangle
$$

where

$$
\left\langle c_{1}[x(t), t] x\left(t^{\prime}\right)\right\rangle=\int d x \int d x^{\prime} x^{\prime} c_{1}(x, t) P_{2}\left(x^{\prime}, t^{\prime} ; x, t\right)
$$

The derivation of (10) shows that it can be generalized as

$$
(\partial / \partial t)\left\langle x(t) f\left[x\left(t^{\prime}\right)\right]\right\rangle=\left\langle c_{1}[x(t), t] f\left[x\left(t^{\prime}\right)\right]\right\rangle, \quad t>t^{\prime}
$$

where $f(x)$ is an arbitrary function. In particular, we have

$$
(\partial / \partial t)\left\langle x(t) c_{1}\left[x\left(t^{\prime}\right), t^{\prime}\right]\right\rangle=\left\langle c_{1}[x(t), t] c_{1}\left[x\left(t^{\prime}\right), t^{\prime}\right]\right\rangle, \quad t>t^{\prime}
$$

which will be needed later.

Higher order two-time correlation functions can be obtained starting from (9). For example, one finds

$$
\begin{aligned}
(\partial / \partial t)\left\langle x^{2}(t) x\left(t^{\prime}\right)\right\rangle= & 2\left\langle c_{1}[x(t), t] x(t) x\left(t^{\prime}\right)\right\rangle \\
& +\epsilon\left\langle c_{2}[x(t), t] x\left(t^{\prime}\right)\right\rangle, \quad t>t^{\prime}
\end{aligned}
$$

which will also be needed later.

In the case of a multivariable description of the macroscopic system, $x(t)$ is replaced by a vector $\mathbf{x}(t)=\operatorname{col}\left[x_{1}(t), \ldots, x_{n}(t)\right]$ in the master equation (3). The components of $\mathbf{x}$ denote different intensive quantities. The moment equations become

$$
\begin{aligned}
d\langle\mathbf{x}(t)\rangle / d t= & \left\langle\mathbf{c}_{1}[\mathbf{x}(t), t]\right\rangle \\
d\langle\mathbf{x}(t) \tilde{\mathbf{x}}(t)\rangle / d t= & \left\langle\mathbf{c}_{1}[\mathbf{x}(t), t] \tilde{\mathbf{x}}(t)\right\rangle+\left\langle\mathbf{x}(t) \tilde{\mathbf{c}}_{1}[\mathbf{x}(t), t]\right\rangle \\
& +\epsilon\left\langle\mathbf{c}_{2}[\mathbf{x}(t), t]\right\rangle \\
(\partial / \partial t)\left\langle\mathbf{x}(t) \tilde{\mathbf{x}}\left(t^{\prime}\right)\right\rangle= & \left\langle\mathbf{c}_{1}[\mathbf{x}(t), t] \tilde{\mathbf{x}}\left(t^{\prime}\right)\right\rangle, \quad t>t^{\prime}
\end{aligned}
$$

where $c_{1}$ is a vector, $c_{2}$ is a square matrix, and $\tilde{\mathbf{x}}$ is the transpose of the column vector $\mathbf{x}$. Other moment equations can be written in a similar way in tensor notation; however, we shall not need them in the subsequent analysis. 


\section{FOUNDATION OF LANGEVIN EOUATION METHOD}

Let $\mathbf{x}(t)$, as a function of time, be a particular realization of the Markoy process. We may formally operate on this function to define a new function $s(t) \equiv s[t, \mathbf{x}(t)]$ as

$$
d \mathbf{x}(t) / d t=\mathbf{c}_{\mathbf{1}}[\mathbf{x}(t), t]+\sqrt{\epsilon} \delta(t)
$$

where the factor $\sqrt{\epsilon}$ is introduced, anticipating the scaling property of $\delta(t)$, to simplify the notation. In this equation, $\mathbf{c}_{1}(\mathbf{x}, t)$ is assumed to be a known function of $\mathbf{x}$ and $t$. The question of the definition and existence of the derivative of the random function $\mathbf{x}(t)$ certainly requires some attention at this point, but we leave this to mathematicians ${ }^{(10)}$ and proceed formally.

The operation in (17) defines a new random process $\{s(t)\}$ whose statistical properties can be obtained in principle from the description of the original Markov process $\{\mathbf{x}(t)\}$. The following properties of $s(t)$ are needed for the present discussion:

$$
\begin{aligned}
\langle\delta(t)\rangle & =0 \\
\left\langle\delta(t) \tilde{\mathbf{x}}\left(t^{\prime}\right)\right\rangle & =0, \quad t>t^{\prime} \\
\langle\delta(t) \tilde{\mathbf{x}}(t)\rangle+\langle\mathbf{x}(t) \tilde{\delta}(t)\rangle & =\sqrt{\epsilon}\left\langle\mathbf{c}_{2}[\mathbf{x}(t), t]\right\rangle \\
\left\langle\delta(t) \tilde{\delta}\left(t^{\prime}\right)\right\rangle & =\left\langle\mathbf{c}_{2}[\mathbf{x}(t), t]\right\rangle \delta\left(t-t^{\prime}\right)
\end{aligned}
$$

The first property is trivially proved by taking the ensemble average of (17) in the sense of (8) and using (14). In fact, the particular operator defined by (17) is chosen so that (18a) holds. Relation (18b) is obtained by multiplying (17) by $\tilde{\mathbf{x}}\left(t^{\prime}\right)$, taking the ensemble average, and comparing the resulting equation to (16). It represents the causality condition. The property (18c) is proved by first multiplying (17) from the right by $\tilde{\mathbf{x}}(t)$, then multiplying its transpose from the left by $\mathbf{x}(t)$, taking the ensemble average of the resulting equations, and finally adding them up. When the final equation is compared to $(15),(18 \mathrm{c})$ is obtained.

The proof of (18d) is somewhat lengthy. Since it plays an important role in the Langevin theory, we present its proof in the one-dimensional case. We essentially foliow a procedure used by $\operatorname{Lax}^{(9)}$ and extend it slightly to the nonlinear nonstationary case. From (17)

$$
\begin{aligned}
\epsilon\left\langle s(t) s\left(t^{\prime}\right)\right\rangle= & \left\langle\left\{\frac{d x(t)}{d t}-c_{1}[x(t), t]\right\}\left\{\frac{d x\left(t^{\prime}\right)}{d t^{\prime}}-c_{1}\left[x\left(t^{\prime}\right), t^{\prime}\right]\right\}\right\rangle \\
= & \frac{\partial^{2}}{\partial t \partial t^{\prime}}\left\langle x(t) x\left(t^{\prime}\right)\right\rangle-\frac{\partial}{\partial t}\left\langle x(t) c_{1}\left[x\left(t^{\prime}\right), t^{\prime}\right]\right\rangle \\
& -\frac{\partial}{\partial t^{\prime}}\left\langle c_{1}[x(t), t] x\left(t^{\prime}\right)\right\rangle+\left\langle c_{1}[x(t), t] c_{1}\left[x\left(t^{\prime}\right), t^{\prime}\right]\right\rangle
\end{aligned}
$$


When $t=t^{\prime}$ the first term in (19) is to be interpreted as

$$
\left.\left(\partial^{2} / \partial t \partial t^{\prime}\right)\left\langle x(t) x\left(t^{\prime}\right)\right\rangle\right|_{t=t^{\prime}}
$$

Let us first consider the case $t \neq t^{\prime}$, and, to be specific, assume $t>t^{\prime}$. Then, from (16)

$$
\left(\partial^{2} / \partial t \partial t^{\prime}\right)\left\langle x(t) x\left(t^{\prime}\right)\right\rangle=\left(\partial / \partial t^{\prime}\right)\left\langle c_{1}[x(t), t] x\left(t^{\prime}\right)\right\rangle
$$

This cancels the third term in (19). The last term cancels the second when $t>t^{\prime}$ by virtue of (12). When $t<t^{\prime}$ one proceeds in a similar way and shows that in this case the first term cancels the second, and so on. Hence, we establish $\left\langle s(t) s\left(t^{\prime}\right)\right\rangle=0$ for $t \neq t^{\prime}$. In order to handle the case $t=t^{\prime}$, we introduce the unit step function $H(t)$ with $H(t)=1, t>0$, and $H(t)+$ $H(-t)=1$, and use the identity

$$
\left\langle x(t) x\left(t^{\prime}\right)\right\rangle=H\left(t-t^{\prime}\right)\left\langle x(t) x\left(t^{\prime}\right)\right\rangle+H\left(t^{\prime}-t\right)\left\langle x(t) x\left(t^{\prime}\right)\right\rangle
$$

One can verify that

$$
\begin{aligned}
& \left(\partial^{2} / \partial t \partial t^{\prime}\right)\left[H\left(t-t^{\prime}\right)\left\langle x(t) x\left(t^{\prime}\right)\right\rangle\right] \\
& =-\delta^{\prime}\left(t-t^{\prime}\right)\langle x(t) x(t)\rangle-\delta\left(t-t^{\prime}\right)\left\langle c_{1}[x(t), t] x(t)\right\rangle \\
& \quad+H\left(t-t^{\prime}\right)\left(\partial / \partial t^{\prime}\right)\left\langle c_{1}[x(t), t] x\left(t^{\prime}\right)\right\rangle
\end{aligned}
$$

where $\delta^{\prime}(t)$ is the derivative of the Dirac delta function $\delta(t)$. In obtaining (20), we used $\delta\left(t-t^{\prime}\right)\left\langle x(t) x\left(t^{\prime}\right)\right\rangle=\delta\left(t-t^{\prime}\right)\langle x(t) x(t)\rangle$ after performing differentiation with respect to $t$. Similarly, one has

$$
\begin{aligned}
\left(\partial^{2} / \partial t \partial t^{\prime}\right)\left[H\left(t^{\prime}-t\right)\left\langle x(t) x\left(t^{\prime}\right)\right\rangle\right] & \\
= & -\delta^{\prime}\left(t^{\prime}-t\right)\left\langle x\left(t^{\prime}\right) x\left(t^{\prime}\right)\right\rangle-\delta\left(t-t^{\prime}\right)\left\langle x(t) c_{1}[x(t), t]\right\rangle \\
& +H\left(t^{\prime}-t\right)(\partial / \partial t)\left\langle x(t) c_{1}\left[x\left(t^{\prime}\right), t^{\prime}\right]\right\rangle
\end{aligned}
$$

Adding (20) and (21) and using $\delta^{\prime}\left(t-t^{\prime}\right)=-\delta^{\prime}\left(t^{\prime}-t\right)$, we obtain

$$
\begin{aligned}
\frac{\partial^{2}}{\partial t \partial t^{\prime}}\langle & \left.x(t) x\left(t^{\prime}\right)\right\rangle \\
= & \delta^{\prime}\left(t-t^{\prime}\right)\left[\left\langle x\left(t^{\prime}\right) x\left(t^{\prime}\right)\right\rangle-\langle x(t) x(t)\rangle\right] \\
& -\delta\left(t-t^{\prime}\right)\left[\left\langle x(t) c_{1}[x(t), t]\right\rangle+\left\langle c_{1}[x(t), t] x(t)\right\rangle\right] \\
& +H\left(t-t^{\prime}\right) \frac{\partial}{\partial t^{\prime}}\left\langle c_{1}[x(t), t] x\left(t^{\prime}\right)\right\rangle+H\left(t^{\prime}-t\right) \frac{\partial}{\partial t}\left\langle x(t) c_{1}\left[x\left(t^{\prime}\right), t^{\prime}\right]\right\rangle
\end{aligned}
$$

The first term in (22a) is simplified by ${ }^{(11)}$

$$
\delta^{\prime}(t) f(t)=-\delta(t) f^{\prime}(0)+f(0) \delta^{\prime}(t)
$$


as

$$
\begin{aligned}
\delta^{\prime}(t & \left.-t^{\prime}\right)\left[\left\langle x\left(t^{\prime}\right) x\left(t^{\prime}\right)\right\rangle-\langle x(t) x(t)\rangle\right] \\
& =\delta^{\prime}(\tau)\left[\left\langle x\left(t^{\prime}\right) x\left(t^{\prime}\right)\right\rangle-\left\langle x\left(t^{\prime}+\tau\right) x\left(t^{\prime}+\tau\right)\right\rangle\right] \\
& =\delta(\tau)(\partial / \partial \tau)\left\langle x\left(t^{\prime}+\tau\right) x\left(t^{\prime}+\tau\right)\right\rangle \\
& =\delta\left(t-t^{\prime}\right)(d / d t)\langle x(t) x(t)\rangle
\end{aligned}
$$

Substituting (22b) into (22a) and the resulting equation into (19), we obtain in matrix form

$$
\begin{aligned}
\epsilon\left\langle\zeta(t) \tilde{S}\left(t^{\prime}\right)\right\rangle= & \delta\left(t-t^{\prime}\right)\left[(d / d t)\langle\mathbf{x}(t) \tilde{\mathbf{x}}(t)\rangle-\left\langle\mathbf{c}_{1}[\mathbf{x}(t), t] \tilde{\mathbf{x}}(t)\right\rangle\right. \\
& \left.-\left\langle\mathbf{x}(t) \tilde{\mathbf{c}}_{1}[\mathbf{x}(t), t]\right\rangle\right]
\end{aligned}
$$

If we eliminate $d[\langle\mathbf{x}(t) \tilde{\mathbf{x}}(t)\rangle] / d t$ in (23) using (15), we get (18d). It is to be noted that these conclusions are valid quite generally for any Markov process. Although we have based the derivations in this section on the master equation (3), written in terms of intensive variables, the same conclusions could be obtained directly, starting from the original master equation (1) for the extensive variables. One simply sets $\epsilon=1$ to obtain the corresponding equations in terms of the extensive variables. The scaling property (2) of the transition probability has not been used up to this point. It becomes essential only when $\epsilon$ is treated as the smallness parameter and quantities are expanded in powers of $\epsilon$ as is discussed below.

When the process is homogeneous in time, the transition probability $\omega$ and its moments (4) are independent of time. If in addition the process is stationary, then all the one-time moments are constant in time. In particular, (15) reduces to

$$
\epsilon\left\langle\mathbf{c}_{2}(\mathbf{x})\right\rangle=-\left[\left\langle\mathbf{c}_{1}(\mathbf{x}) \tilde{\mathbf{x}}\right\rangle+\left\langle\mathbf{x} \tilde{\mathbf{c}}_{1}(\mathbf{x})\right\rangle\right]
$$

which is the generalized Einstein relation ${ }^{(9)}$ for a nonlinear process.

The physical implication of Eq. (17) is that it enables one to interpret the fluctuations of the macroscopic variables as the response of a nonlinear timevarying system described by a deterministic equation

$$
d \mathbf{x}(t) / d t=\mathbf{c}_{1}[\mathbf{x}(t), t]
$$

to a random source $\sqrt{\epsilon} \delta(t) .^{2}$ Equation (17), which is the Langevin equation for a nonlinear system, provides an alternative and formally equivalent description of the original random process $\{\mathbf{x}(t)\}$ in terms of the source process $\{\delta(t)\}$. The usefulness of this description lies in the fact that it lends

${ }^{2}$ This interpretation is usually considered as the "Langevin assumption," (5) but actually it is not an assumption until one specifies $\delta(t)$. 
itself well to approximations, especially if only the first two moments and the correlation function of the fluctuations are of interest. The system-size expansion provides a systematic procedure to implement these approximations.

\section{SYSTEM-SIZE EXPANSION OF THE LANGEVIN EQUATION}

To avoid cumbersome notation, we present the derivation of the approximate Langevin equations in the one-dimensional case, and start with

$$
d x / d t=c_{1}(x)+\sqrt{\epsilon} \delta
$$

where we have suppressed the arguments of $x(t)$ and $s(t)$ and the explicit time dependence of $c_{1}(x, t)$ to compress the formulas. The form of $(25)$ and the fact that $\langle\delta(t)\rangle=0$ suggest the following substitution:

$$
x=\langle x\rangle+\sqrt{\epsilon} \xi
$$

where $\langle x(t)\rangle$ is the mean and $\xi(t)$ is the fluctuation about the mean, viz. $\langle\xi(t)\rangle=0$. The equations for $\langle x(t)\rangle$ and $\xi(t)$ follow from (25) as

$$
\begin{aligned}
d\langle x\rangle / d t= & c_{1}(\langle x\rangle)+\frac{1}{2} \epsilon c_{1}^{\prime \prime}(\langle x\rangle)\left\langle\xi^{2}\right\rangle+\frac{1}{6} \epsilon^{3 / 2} c_{1}^{\prime \prime \prime}(\langle x\rangle)\left\langle\xi^{3}\right\rangle+O\left(\epsilon^{2}\right) \\
d \xi / d t= & c_{1}{ }^{\prime}(\langle x\rangle) \xi+\frac{1}{2} \epsilon^{1 / 2} c_{1}^{\prime \prime}(\langle x\rangle)\left[\xi^{2}-\left\langle\xi^{2}\right\rangle\right] \\
& +\frac{1}{6} \epsilon \mathcal{c}_{1}^{\prime \prime \prime}(\langle x\rangle)\left[\xi^{3}-\left\langle\xi^{3}\right\rangle\right]+\varsigma(t)+O\left(\epsilon^{3 / 2}\right)
\end{aligned}
$$

where $c_{1}{ }^{\prime}, c_{1}^{\prime \prime}, \ldots$ denote the partial derivatives of $c_{1}(x, t)$ with respect to $x$. Since the lowest power of $\epsilon$ is $\epsilon$ in (27) and $\epsilon^{1 / 2}$ in (28), we expand $\langle x\rangle$ and $\xi$ as

$$
\begin{aligned}
\langle x\rangle & =y+\epsilon u_{0}+\epsilon^{3 / 2} u_{1} \\
\xi & =\xi_{0}+\sqrt{\epsilon} \xi_{1}+\epsilon \xi_{2}
\end{aligned}
$$

where $y(t)$ is the mean, and $\xi_{0}(t)$ is the fluctuation about the mean in the lowest order in $\epsilon$, both being independent of $\epsilon$. Similarly the terms of the next order $u_{0}(t)$ and $\xi_{1}(t)$ also do not depend on $\epsilon$ explicitly. But $u_{1}(t)$ and $\xi_{2}(t)$ must depend on $\epsilon$ implicitly because they account for the rest of the terms in a power series expansion in $\epsilon^{1 / 2}$. They are of order $\epsilon^{0}$ in the sense that they do not vanish as $\epsilon \rightarrow 0$. Substituting (29) and (30) into (27) and (28), respectively, we obtain

$$
\begin{aligned}
d y / d t & =c_{1}(y) \\
d u_{0} / d t & =u_{0} c_{1}^{\prime}(y)+\frac{1}{2} c_{1}^{\prime \prime}(y)\left\langle\xi_{0}^{2}\right\rangle \\
d u_{1} / d t & =u_{1} c_{1}^{\prime}(y)+c_{1}^{\prime \prime}(y)\left\langle\xi_{0} \xi_{1}\right\rangle+\frac{1}{6} c_{1}^{\prime \prime}(y)\left\langle\xi_{0}^{3}\right\rangle+O\left(\epsilon^{1 / 2}\right)
\end{aligned}
$$


for the evolution of the mean, and

$$
\begin{aligned}
d \xi_{0} / d t= & \xi_{0} c_{1}^{\prime}(y)+\delta_{0}(t) \\
d \xi_{1} / d t= & \xi_{1} c_{1}^{\prime}(y)+\frac{1}{2} c_{1}^{\prime \prime}(y)\left[\xi_{0}^{2}-\left\langle\xi_{0}^{2}\right\rangle\right]+\diamond_{1}(t) \\
d \xi_{2} / d t= & \xi_{2} c_{1}^{\prime}(y)+u_{0} \xi_{0} c_{1}^{\prime \prime}(y)+c_{1}^{\prime \prime}(y)\left[\xi_{0} \xi_{1}-\left\langle\xi_{0} \xi_{1}\right\rangle\right] \\
& +\frac{1}{6} c_{1}^{\prime \prime \prime}(y)\left[\xi_{0}{ }^{3}-\left\langle\xi_{0}{ }^{3}\right\rangle\right]+\delta_{2}(t)+O\left(\epsilon^{1 / 2}\right)
\end{aligned}
$$

for the evolution of the fluctuations. Equations (34)-(36) constitute the approximate set of Langevin equations ${ }^{3}$ with different random sources $s_{0}(t)$, $s_{1}(t)$, and $s_{2}(t)$. The latter are introduced by expanding $s(t)$ in (28) as

$$
\delta(t)=\delta_{0}(t)+\sqrt{\epsilon} \delta_{1}(t)+\epsilon \delta_{2}(t)
$$

Here also $\delta_{0}(t)$ and $\delta_{1}(t)$ are independent of $\epsilon$, but $\delta_{2}(t)$ depends on $\epsilon$ implicitly. The properties of these sources are obtained from the relations (18) satisfied by $\delta(t), x(t)$, and $c_{2}(x, t)$ by substituting $x=y+\sqrt{\epsilon} \xi_{0}+$ $\epsilon\left(u_{0}+\xi_{1}\right)+\epsilon^{3 / 2}\left(u_{1}+\xi_{2}\right)$ and expanding $c_{2}(x, t)$ about $y$. We present the results only ${ }^{4}$ :

$$
\begin{aligned}
\left\langle\delta_{0}(t)\right\rangle & =0 \\
\left\langle\delta_{0}(t) \xi_{0}\left(t^{\prime}\right)\right\rangle= & 0, \quad t>t_{1} \\
\left\langle\delta_{0}(t) \xi_{0}(t)\right\rangle= & \frac{1}{2} c_{2}(y) \\
\left\langle\delta_{0}(t) s_{0}\left(t^{\prime}\right)\right\rangle= & c_{2}(y) \delta\left(t-t^{\prime}\right) \\
\left\langle\delta_{1}(t)\right\rangle= & 0 \\
\left\langle\delta_{1}(t) \xi_{1}\left(t^{\prime}\right)\right\rangle= & 0, \quad t>t^{\prime} \\
\left\langle\delta_{1}(t) \xi_{1}(t)\right\rangle= & \frac{1}{2}\left[c_{2}^{\prime}(y) u_{0}+\frac{1}{2} c_{2}^{\prime \prime}(y)\left\langle\xi_{0}{ }^{2}\right\rangle\right] \\
\left\langle\delta_{1}(t) \delta_{1}\left(t^{\prime}\right)\right\rangle= & {\left[c_{2}{ }^{\prime}(y) u_{0}+\frac{1}{2} c_{2}^{\prime \prime}(y)\left\langle\xi_{0}{ }^{2}\right\rangle\right] \delta\left(t-t^{\prime}\right) } \\
\left\langle\delta_{2}(t)\right\rangle= & 0 \\
\left\langle\delta_{2}(t) \xi_{2}\left(t^{\prime}\right)\right\rangle= & 0, \quad t>t^{\prime} \\
\left\langle\delta_{2}(t) \xi_{2}(t)\right\rangle= & \frac{1}{4}\left[u_{0}{ }^{2} c_{2}^{\prime \prime}(y)+u_{0}\left\langle\xi_{0}{ }^{2}\right\rangle c_{2}^{\prime \prime \prime}(y)+\left\langle\xi_{0}{ }^{2} \xi_{1}\right\rangle c_{2}^{\prime \prime \prime}(y)\right. \\
& \left.+c_{2}^{\prime \prime}(y)\left\langle\xi_{1}{ }^{2}\right\rangle\right] \\
\left\langle\delta_{2}(t) \delta_{2}\left(t^{\prime}\right)\right\rangle= & 2\left\langle\delta_{2}(t) \xi_{2}(t)\right\rangle \delta\left(t-t^{\prime}\right)
\end{aligned}
$$

${ }^{3}$ The terms proportional to $\left\langle\xi_{0}{ }^{3}\right\rangle$ are actually not present in (33) and (36) because $\xi_{0}(t)$ is Gaussian at all times, as shown later.

${ }^{4}$ Actually in place of (39b) one obtains

$$
\left\langle\delta_{0}(t) \xi_{2}\left(t^{\prime}\right)\right\rangle+\left\langle\delta_{2}(t) \xi_{0}\left(t^{\prime}\right)\right\rangle+\left\langle\delta_{1}(t) \xi_{1}\left(t^{\prime}\right)\right\rangle=0, \quad t>t^{\prime}
$$

but it seems that one can split this into (39b) and (42b) without violating any consistency relation. However, only this combined form appears in the later derivations. 
Equations (38)-(40) are the usual properties satisfied by the Langevin sources. In addition to these, we also get the following cross-correlations:

$$
\begin{aligned}
\left\langle\delta_{0}(t) \delta_{1}\left(t^{\prime}\right)\right\rangle+\left\langle\delta_{0}\left(t^{\prime}\right) s_{1}(t)\right\rangle= & 0 \\
\left\langle\delta_{0}(t) \delta_{2}\left(t^{\prime}\right)\right\rangle+\left\langle\delta_{0}\left(t^{\prime}\right) \delta_{2}(t)\right\rangle= & 0 \\
\left\langle\delta_{1}(t) \delta_{2}\left(t^{\prime}\right)\right\rangle+\left\langle\delta_{1}\left(t^{\prime}\right) \delta_{2}(t)\right\rangle= & {\left[c_{2}{ }^{\prime}(y) u_{1}+c_{2}^{\prime \prime}(y)\left\langle\xi_{0} \xi_{1}\right\rangle\right.} \\
& \left.+\frac{1}{6} c_{2}^{\prime \prime \prime}(y)\left\langle\xi_{0}{ }^{3}\right\rangle\right] \delta\left(t-t^{\prime}\right) \\
\left\langle\Delta_{1}(t) \xi_{0}\left(t^{\prime}\right)\right\rangle+\left\langle\delta_{0}(t) \xi_{1}\left(t^{\prime}\right)\right\rangle= & 0, \quad t \geqslant t^{\prime} \\
\left\langle\Delta_{0}(t) \xi_{2}\left(t^{\prime}\right)\right\rangle+\left\langle\delta_{2}(t) \xi_{0}\left(t^{\prime}\right)\right\rangle= & 0, \quad t \geqslant t^{\prime} \\
\left\langle\delta_{1}(t) \xi_{2}\left(t^{\prime}\right)\right\rangle+\left\langle\delta_{2}(t) \xi_{1}\left(t^{\prime}\right)\right\rangle= & 0, \quad t>t^{\prime} \\
\left\langle\delta_{1}(t) \xi_{2}(t)\right\rangle+\left\langle\delta_{2}(t) \xi_{1}(t)\right\rangle= & \frac{1}{2}\left[c_{2}{ }^{\prime}(y) u_{1}+c_{2}^{\prime \prime}(y)\left\langle\xi_{0} \xi_{1}\right\rangle\right. \\
& \left.+\frac{1}{6} c_{2}^{\prime \prime \prime}(y)\left\langle\xi_{0}{ }^{3}\right\rangle\right]
\end{aligned}
$$

Equations (4la) and (4lb) may be interpreted as denoting that the random source $\delta_{0}(t)$ is uncorrelated with $\delta_{1}(t)$ and $\delta_{2}(t)$, although the system-size expansion requires only (41a) and (4lb) to hold.

We can obtain other higher order correlations involving the random sources and fluctuations. We present some of the relations that are used in the applications discussed later. Multiplying the Langevin equation (25) by $3 x^{2}(t)$ and taking ensemble averages, we obtain

$$
d\left\langle x^{3}\right\rangle / d t=3\left\langle c_{1}(x) x^{2}\right\rangle+3 \sqrt{\epsilon}\left\langle\delta(t) x^{2}(t)\right\rangle
$$

A comparison of this equation to (7) leads to

$$
\left\langle\delta(t) x^{2}(t)\right\rangle=\sqrt{\epsilon}\left\langle c_{2}(x) x\right\rangle+\frac{1}{3} \epsilon^{3 / 2}\left\langle c_{3}(x)\right\rangle
$$

Substituting $x=\langle x\rangle+\sqrt{\epsilon} \xi$ in (44), we get

$$
\left\langle s(t) \xi^{2}(t)\right\rangle=\left\langle c_{2}(x) \xi\right\rangle+\frac{1}{3} \sqrt{\epsilon}\left\langle c_{3}(x)\right\rangle
$$

where we have used

$$
\langle s(t) \xi(t)\rangle=\frac{1}{2}\left\langle c_{2}(x)\right\rangle
$$

Expanding $s(t), \xi(t)$, and $x(t)$ in powers of $\epsilon$, we obtain

$$
\begin{aligned}
\left\langle s_{0}(t) \xi_{0}{ }^{2}(t)\right\rangle & =0 \\
2\left\langle s_{0}(t) \xi_{0}(t) \xi_{1}(t)\right\rangle+\left\langle s_{1}(t) \xi_{0}{ }^{2}(t)\right\rangle & =c_{2}{ }^{\prime}(y)\left\langle\xi_{0}{ }^{2}\right\rangle+\frac{1}{3} c_{3}(y)
\end{aligned}
$$

Equation (46) is also a consequence of the Gaussian nature of $\xi_{0}(t)$, which will be proven later. 
We now multiply the Langevin equation (25) by $2 x(t) x\left(t^{\prime}\right), t>t^{\prime}$, and take the ensemble averages to obtain

$$
\begin{aligned}
(\partial / \partial t)\left\langle x^{2}(t) x\left(t^{\prime}\right)\right\rangle= & \left.2\left\langle c_{1}[x(t)] x(t) x\left(t^{\prime}\right)\right]\right\rangle \\
& +\sqrt{\epsilon} 2\left\langle\delta(t) x(t) x\left(t^{\prime}\right)\right\rangle, \quad t>t^{\prime}
\end{aligned}
$$

Comparing this equation to (13) yields

$$
\left\langle s(t) x(t) x\left(t^{\prime}\right)\right\rangle=\frac{1}{2} \sqrt{\epsilon}\left\langle c_{2}[x(t)] x\left(t^{\prime}\right)\right\rangle, \quad t>t^{\prime}
$$

which can be reduced to

$$
\left\langle\delta(t) \xi(t) \xi\left(t^{\prime}\right)\right\rangle=\frac{1}{2}\left\langle c_{2}[x(t)] \xi\left(t^{\prime}\right)\right\rangle, \quad t>t^{\prime}
$$

with $x=\langle x\rangle+\sqrt{\epsilon} \xi(t)$. Again expanding $x$, $\xi$, and $s$ as in (29), (30), and (37), we obtain

$$
\begin{gathered}
\left\langle\Delta_{0}(t) \xi_{0}(t) \xi_{0}\left(t^{\prime}\right)\right\rangle=0, \quad t>t^{\prime} \\
\left\langle s_{1}(t) \xi_{0}(t) \xi_{0}\left(t^{\prime}\right)\right\rangle+\left\langle\Delta_{0}(t) \xi_{1}(t) \xi_{0}\left(t^{\prime}\right)\right\rangle+\left\langle b_{0}(t) \xi_{0}(t) \xi_{1}\left(t^{\prime}\right)\right\rangle \\
=\frac{1}{2} c_{2}^{\prime}(y)\left\langle\xi_{0}(t) \xi_{0}\left(t^{\prime}\right)\right\rangle, \quad t>t^{\prime}
\end{gathered}
$$

The conclusion (50) is also a consequence of the Gaussian nature of $\xi_{0}(t)$ and $\delta_{0}(t)$. Equation (51) will be used in constructing the power spectral density correct to order $\epsilon^{2}$ in Section 6.

\section{LANGEVIN DESCRIPTION IN THE LOWEST ORDER IN $\epsilon$}

We summarize the Langevin equations in the lowest order in $\epsilon$ :

$$
\begin{aligned}
d y / d t & =c_{1}(y, t) \\
d \xi_{0} / d t & =\xi_{0} c_{1}{ }^{\prime}(y, t)+\varsigma_{0}(t)
\end{aligned}
$$

where $s_{0}(t)$ satisfies Eqs. (38). Equation (52) determines the evolution of the mean $\langle x(t)\rangle=y(t)+\epsilon u_{0}(t)+\epsilon^{3 / 2} u_{1}(t)+O\left(\epsilon^{2}\right)$ in the limit $\epsilon \rightarrow 0$, and is referred to by Kubo et $a l^{(2)}$ as the deterministic equation. In the timeinvariant system, $c_{1}(y, t)$ [as well as $c_{2}(y, t)$ in (38)] does not depend on time explicitly. In this case the solution of (52) can always be constructed by quadrature $^{(1)}$ :

$$
t-t_{0}=\int_{y_{0}}^{y} d y^{\prime} \frac{1}{c_{1}\left(y^{\prime}\right)}
$$

where $y_{0}$ is the initial condition at $t_{0}$.

The stochastic equation (53) describes the fluctuations in the lowest 
order, viz., $\xi(t)=\xi_{0}(t)+\epsilon^{1 / 2} \xi_{1}(t)+\epsilon \xi_{2}(t)+O\left(\epsilon^{3 / 2}\right)$. Multiplying it by $2 \xi_{0}(t)$ and using $(38 \mathrm{c})$ yields

$$
d \sigma / d t=2 c_{1}^{\prime}(y, t) \sigma(t)+c_{2}(y, t)
$$

where $\sigma(t)=\left\langle\xi_{0}^{2}\right\rangle$, so that the variance of $x$ is $\epsilon \sigma(t)$. In a time-invariant system its solution can be obtained as

$$
\sigma(y)=\sigma_{0}\left[c_{1}(y) / c_{1}\left(y_{0}\right)\right]^{2}+c_{1}^{2}(y) \int_{y_{0}}^{y} d y^{\prime} c_{2}\left(y^{\prime}\right) / c_{1}^{3}\left(y^{\prime}\right)
$$

where $\sigma_{0}$ is the initial value of $\sigma(t)$ at $t_{0}$. This result was obtained by van Kampen ${ }^{(1)}$ and Kubo et $a l^{(2)}$ As pointed out by the latter authors, $\sigma(t)$ is determined by the value of $y$ at that instant in the case of a single variable, as demonstrated by (56).

We can obtain the autocorrelation of $x(t)$, i.e.,

$$
\begin{aligned}
\Phi\left(t, t^{\prime}\right) & =\left\langle[x(t)-\langle x(t)\rangle]\left[x\left(t^{\prime}\right)-\left\langle x\left(t^{\prime}\right)\right\rangle\right]\right\rangle \\
& =\epsilon\left\langle\xi(t) \xi\left(t^{\prime}\right)\right\rangle=\epsilon\left\langle\xi_{0}(t) \xi_{0}\left(t^{\prime}\right)\right\rangle+O\left(\epsilon^{2}\right)
\end{aligned}
$$

in the lowest order from the Langevin equation (53) by multiplying it by $\xi_{0}\left(t^{\prime}\right)$, taking the ensemble average, and using (38b), as

$$
\partial \Phi\left(t, t^{\prime}\right) / \partial t=c_{1}^{\prime}(y, t) \Phi\left(t, t^{\prime}\right), \quad t>t^{\prime}
$$

whose solution is

$$
\Phi\left(t, t^{\prime}\right)=\exp \left\{\int_{t^{\prime}}^{t} d u c_{1}^{\prime}[y(u), u]\right\} \phi\left(t^{\prime}, t^{\prime}\right), \quad t>t^{\prime}
$$

In the case of a time-invariant system, (59) can be expressed in a more compact form by noting that $\Phi\left(t^{\prime}, t^{\prime}\right)=\epsilon \sigma\left(t^{\prime}\right)$ and expressing the integral in the exponent as

$$
\int_{t^{\prime}}^{t} d u c_{1}^{\prime}[y(u)]=\int_{y\left(t^{\prime}\right)}^{y(t)} d \ln c_{1}(y)
$$

with the help of (52). The result is

$$
\Phi\left(t, t^{\prime}\right)=\epsilon \sigma\left(t^{\prime}\right) c_{1}[y(t)] / c_{1}\left[y\left(t^{\prime}\right)\right], \quad t>t^{\prime}
$$

where $y(t)$ is given by (54) with $t>t^{\prime}>t_{0}$. This result was also obtained by Kubo et al. ${ }^{(2)}$ by a different method. In the following section we evaluate $\Phi\left(t, t^{\prime}\right)$ when the system is in a stable equilibrium state, correct to order $\epsilon^{2}$.

We can obtain the deviation of the mean from the deterministic path in the lowest order once the variance $\sigma(t)$ is determined from (55), using (32):

$$
d u_{0} / d t=u_{0} c_{1}^{\prime}(y, t)+\frac{1}{2} \sigma(t) c_{1}^{\prime \prime}(y, t)
$$


The evolution of the mean is then obtained as

$$
\langle x(t)\rangle=y(t)+\epsilon u_{0}(t)+O\left(\epsilon^{3 / 2}\right)
$$

It is noted that the lowest order description of the fluctuations through the Langevin equation enables one to determine the mean correct to order $\epsilon$.

\subsection{Fokker-Planck Equation}

The Langevin equation (53) does not provide the distribution function $\Pi\left(\xi_{0}, t\right)$ of $\xi_{0}$. However, if $\delta_{0}(t)$ were Gaussian, it would have led to the following Fokker-Planck equation ${ }^{(4)}$ :

$$
\frac{\partial \Pi\left(\xi_{0}, t\right)}{\partial t}=-c_{1}{ }^{\prime}(y, t) \frac{\partial}{\partial \xi_{0}}\left[\xi_{0} \Pi\right]+\frac{1}{2} c_{2}(y, t) \frac{\partial^{2} \Pi}{\partial \xi_{0}}
$$

But this is identical to the Fokker-Planck equation obtained originally by van Kampen ${ }^{(1)}$ in the limit of $\epsilon \rightarrow 0$, using Kramers-Moyal expansion, for the fluctuations about the deterministic path. Since $\langle x\rangle=y+\epsilon \mathcal{U}_{0}+O\left(\epsilon^{3 / 2}\right)$ and $x=\langle x\rangle+\sqrt{\epsilon}\left(\xi_{0}+\sqrt{\epsilon} \xi_{1}\right)+O\left(\epsilon^{3 / 2}\right), \xi_{0}$ reduces to the fluctuations about the deterministic path $y(t)$ as $\epsilon \rightarrow 0$. We therefore conclude that the random source $S_{0}(t)$ and $\xi_{0}(t)$ are Gaussian random processes (cf. footnote 3 ). The non-Gaussian nature of the fluctuations about the mean is accounted for by the higher order fluctuations $\xi_{1}(t), \xi_{2}(t), \ldots$, which satisfy higher order Langevin equations (35), (36),... with their non-Gaussian Langevin sources $\delta_{1}(t), \delta_{2}(t), \ldots$

\subsection{Fluctuations About an Equilibrium State}

The equilibrium state $y_{e}$ of a time-invariant nonlinear system is determined as one of the roots of

$$
c_{1}\left(y_{e}\right)=0
$$

The behavior of the deterministic path about an equilibrium state is governed by

where

$$
d \delta y / d t=\gamma_{1} \delta y
$$

$$
\gamma_{1} \equiv c_{1}^{\prime}\left(y_{e}\right)
$$

If $\gamma_{1}<0$ the equilibrium is stable; otherwise it is unstable. If $\gamma_{1}=0$ but

$$
\gamma_{2} \equiv c_{1}^{\prime \prime}\left(y_{e}\right) \neq 0
$$

then it is a marginal equilibrium. Kubo et al ${ }^{(2)}$ refer to the case $\gamma_{1}=0$, $\gamma_{2}=0$, and

$$
\gamma_{3} \equiv c_{1}^{\prime \prime}\left(y_{e}\right) \neq 0
$$

as the critical equilibrium. Here we restrict our attention to a stable equilibrium. 
Replacing $c_{1}{ }^{\prime}(y, t)$ by $\gamma_{1}$, in (58) we obtain

$$
\Phi\left(t, t^{\prime}\right)=\epsilon\left\{\exp \left[\gamma_{1}\left(t-t^{\prime}\right)\right]\right\} \sigma_{e}, \quad t>t^{\prime}
$$

where $\sigma_{e}$ is determined from (55) as

$$
\sigma_{e}=c_{2}\left(y_{e}\right) / 2\left|\gamma_{1}\right|
$$

The power-spectral density of the fluctuations follows from (68) as

$$
G(\omega)=\epsilon c_{2}\left(y_{e}\right) /\left(\omega^{2}+\gamma_{1}{ }^{2}\right)
$$

The mean value of the fluctuations in a stable equilibrium state is obtained from (61) with $\dot{u}_{0}=0$ as

$$
\langle x\rangle_{\mathrm{eq}}=y_{e}+\epsilon\left(\gamma_{2} / 2\left|\gamma_{1}\right|\right) \sigma_{e}
$$

\subsection{Multivariable Description}

The Langevin equations in the lowest order in $\epsilon$ can easily be written in matrix form when the states of the macroscopic system are characterized by a set of variables $\mathbf{x}(t)=\operatorname{col}\left[x_{1}(t), \ldots, x_{n}(t)\right]$. Defining $\mathbf{x}(t)=\langle\mathbf{x}\rangle+\sqrt{\epsilon} \xi_{0}$ and $\langle\mathbf{x}\rangle=\mathbf{y}+\epsilon \mathbf{u}_{0}$, we find

$$
\begin{aligned}
d \mathbf{y} / d t & =\mathbf{c}_{1}[\mathbf{y}, t] \\
d \xi_{0} / d t & =\wedge[\mathbf{y}, t] \xi_{0}+\swarrow_{0}(t)
\end{aligned}
$$

where

$$
\Lambda[\mathbf{x}, t] \equiv \partial \mathbf{c}_{1}[\mathbf{x}, t] / \partial \mathbf{x}
$$

The Langevin source now satisfies [cf. Eqs. (18)]

$$
\begin{aligned}
\left\langle\delta_{0}(t)\right\rangle & =0 \\
\left\langle\delta_{0}(t) \tilde{\xi}_{0}\left(t^{\prime}\right)\right\rangle & =0, \quad t>t^{\prime} \\
\left\langle\delta_{0}(t) \tilde{\xi}_{0}(t)\right\rangle+\left\langle\xi_{0}(t) \tilde{J}_{0}(t)\right\rangle & =\mathrm{c}_{2}[\mathbf{y}(t), t] \\
\left\langle\delta_{0}(t) \tilde{\xi}_{0}\left(t^{\prime}\right)\right\rangle & =\mathrm{c}_{2}[y(t), t] \delta\left(t-t^{\prime}\right)
\end{aligned}
$$

The evolution of the variance, defined by

$$
\sigma(t)=\left\langle\xi_{0}(t) \tilde{\xi}_{0}(t)\right\rangle
$$

is obtained from (73) as

$$
d \sigma(t) / d t=\Lambda[\mathbf{y}, t] \sigma+\sigma \tilde{\Lambda}[\mathbf{y}, t]+\mathrm{c}_{2}[\mathbf{y}, t]
$$

The power spectral density of the fluctuations about a stable equilibrium state in a time-invariant system is obtained from (73) and (75) as

$$
\mathrm{G}(\omega)=\epsilon(i \omega+\Lambda)^{-1} c_{2}\left(y_{e}\right)(-i \omega \cdot+\tilde{\Lambda})^{-1}
$$


or equivalently

$$
\mathrm{G}(\omega)=\epsilon\left[(i \omega+\Lambda)^{-1} \sigma_{e}+\sigma_{e}(-i \omega+\tilde{\Lambda})^{-1}\right]
$$

as shown by $\operatorname{Lax}^{(12)}$ in connection with the Langevin method in linear systems. Here $\sigma_{e}$ is the equilibrium variance matrix.

\section{HIGHER ORDER LANGEVIN EQUATIONS}

In this section we use higher order Langevin equations to calculate the power spectral density of fluctuations about a stable equilibrium state. For simplicity in notation we restrict ourselves to one variable again. The correlation function in an equilibrium state has the following expansion [cf. (30)]:

$$
\begin{aligned}
\Phi(\tau)= & \epsilon\left\{\left\langle\xi_{0}(\tau) \xi_{0}(0)\right\rangle+\epsilon\left[\left\langle\xi_{1}(\tau) \xi_{1}(0)\right\rangle+\left\langle\xi_{0}(\tau) \xi_{2}(0)\right\rangle\right.\right. \\
& \left.\left.+\left\langle\xi_{2}(\tau) \xi_{0}(0)\right\rangle\right]+\epsilon^{2}\left\langle\xi_{2}(\tau) \xi_{2}(0)\right\rangle\right\}
\end{aligned}
$$

The cross terms proportional to $\sqrt{\epsilon}$ and $\epsilon^{3 / 2}$ in (79) are not included because they vanish in equilibrium, viz.,

$$
\begin{aligned}
& \left\langle\xi_{0}(\tau) \xi_{1}(0)\right\rangle+\left\langle\xi_{1}(\tau) \xi_{0}(0)\right\rangle=0 \\
& \left\langle\xi_{1}(\tau) \xi_{2}(0)\right\rangle+\left\langle\xi_{2}(\tau) \xi_{1}(0)\right\rangle=0
\end{aligned}
$$

The proof of (80a) is presented in the appendix. We do not consider (80b) any further because it is of higher order in $\epsilon$ than that to which we intend to calculate $\Phi(\tau)$. From (79) we find

$$
\Phi(\tau)=\epsilon\left[\left\langle\xi_{0}(\tau) \xi_{0}(0)\right\rangle+\epsilon \varphi(\tau)+O\left(\epsilon^{2}\right)\right]
$$

where

$$
\varphi(\tau) \equiv\left\langle\xi_{1}(\tau) \xi_{1}(0)\right\rangle+\left\langle\xi_{0}(\tau) \xi_{2}(0)\right\rangle+\left\langle\xi_{2}(\tau) \xi_{0}(0)\right\rangle
$$

The first term in (81) was already obtained in the previous section [cf. (68)]. Here we determine $\varphi(\tau)$. The relevant Langevin equations are obtained from (34)-(36) with $y \equiv y_{e}$, where $y_{e}$ is the stable equilibrium state under consideration:

$$
\begin{aligned}
& d \xi_{0} / d t=\gamma_{1} \xi_{0}+\delta_{0} \\
& d \xi_{1} / d t=\gamma_{1} \xi_{1}+\frac{1}{2} \gamma_{2}\left[\xi_{0}^{2}-\left\langle\xi_{0}^{2}\right\rangle\right]+\diamond_{1} \\
& d \xi_{2} / d t=\gamma_{1} \xi_{2}+\gamma_{2} \xi_{0}\left(u_{0 e}+\xi_{1}\right)+\frac{1}{6} \gamma_{3} \xi_{0}{ }^{3}+\jmath_{2}
\end{aligned}
$$

where we have set $\left\langle\xi_{0}{ }^{3}\right\rangle=0$ because $\xi_{0}$ is Gaussian (cf. footnote 3), and $\left\langle\xi_{0} \xi_{1}\right\rangle=0$ by virtue of (80a) (cf. the appendix). We multiply (83) by $\xi_{2}(0)$, 
(84) by $\xi_{1}(0)$, and (85) by $\xi_{0}(0)$, take ensemble averages, add the resulting equations, and obtain for $\tau>0$

$$
\begin{aligned}
d \varphi(\tau) / d \tau-\gamma_{1} \varphi(\tau)= & \frac{1}{2} \gamma_{2}\left\langle\xi_{0}{ }^{2}(\tau) \xi_{1}(0)\right\rangle+u_{0} \gamma_{2}\left\langle\xi_{0}(\tau) \xi_{0}(0)\right\rangle \\
& +\gamma_{2}\left\langle\xi_{0}(\tau) \xi_{1}(\tau) \xi_{0}(0)\right\rangle+\frac{1}{6} \gamma_{3}\left\langle\xi_{0}{ }^{3}(\tau) \xi_{0}(0)\right\rangle
\end{aligned}
$$

where we replaced $t$ by $\tau$, and used [cf. (39b), (42b), and footnote 4]

$$
\left\langle\boldsymbol{s}_{0}(\tau) \xi_{2}(0)\right\rangle+\left\langle\boldsymbol{\delta}_{2}(\tau) \xi_{0}(0)\right\rangle+\left\langle\boldsymbol{s}_{1}(\tau) \xi_{1}(0)\right\rangle=0
$$

Since $\xi_{0}(\tau)$ is a Gaussian process, the last term in (86) can be evaluated as

$$
\left\langle\xi_{0}{ }^{3}(\tau) \xi_{0}(0)\right\rangle=3\left\langle\xi_{0}{ }^{2}\right\rangle\left\langle\xi_{0}(\tau) \xi_{0}(0)\right\rangle
$$

where we use

$$
\left\langle g_{1} g_{2} g_{3} g_{4}\right\rangle=\left\langle g_{1} g_{2}\right\rangle\left\langle g_{3} g_{4}\right\rangle+\left\langle g_{1} g_{3}\right\rangle\left\langle g_{2} g_{4}\right\rangle+\left\langle g_{1} g_{4}\right\rangle\left\langle g_{2} g_{3}\right\rangle
$$

for a Gaussian multivariate.

The $u_{0 e}$ in (85) is obtained from (61) with $\dot{u}_{0 e}=0$ as

$$
u_{0 e}=-\left(\gamma_{2} / 2 \gamma_{1}\right)\left\langle\xi_{0}^{2}\right\rangle
$$

Substitution of (88) and (87) in (86) yields

$$
d \varphi / d \tau-\gamma_{1} \varphi=a_{1}\left\langle\xi_{0}(\tau) \xi_{0}(0)\right\rangle+\gamma_{2} Z(\tau), \quad \tau>0
$$

where we have defined

$$
\begin{aligned}
a_{1} & \equiv \frac{1}{2}\left\langle\xi_{0}{ }^{2}\right\rangle\left[\gamma_{3}-\left(\gamma_{2}{ }^{2} / \gamma_{1}\right)\right] \\
Z(\tau) & \equiv\left\langle\xi_{0}(\tau) \xi_{1}(\tau) \xi_{0}(0)\right\rangle+\frac{1}{2}\left\langle\xi_{0}{ }^{2}(\tau) \xi_{1}(0)\right\rangle
\end{aligned}
$$

By differentiating $Z(\tau)$ with respect to $\tau$ and eliminating the derivatives of $\xi_{0}$ and $\xi_{1}$ using (83) and (84), one gets

$$
\begin{aligned}
d Z / d \tau-2 \gamma_{1} Z= & \frac{1}{2} \gamma_{2}\left[\left\langle\xi_{0}{ }^{3}(\tau) \xi_{0}(0)\right\rangle-\left\langle\xi_{0}{ }^{2}\right\rangle\left\langle\xi_{0}(\tau) \xi_{0}(0)\right\rangle\right] \\
& +\left\langle\delta_{0}(\tau) \xi_{1}(\tau) \xi_{0}(0)\right\rangle+\left\langle\delta_{0}(\tau) \xi_{0}(\tau) \xi_{1}(0)\right\rangle \\
& +\left\langle\delta_{1}(\tau) \xi_{0}(\tau) \xi_{0}(0)\right\rangle, \quad \tau>0
\end{aligned}
$$

The first term in (92) is already calculated in (87). The last three terms are given by (51), so that (92) reduces to

$$
d Z / d \tau-2 \gamma_{1} Z=\left(\gamma_{2}+\frac{1}{2} c_{2}{ }^{\prime}\right)\left\langle\xi_{0}(\tau) \xi_{0}(0)\right\rangle, \quad \tau>0
$$

where $c_{2}{ }^{\prime}=c_{2}{ }^{\prime}\left(y_{e}\right)$. One needs

$$
Z(0) \equiv \frac{3}{2}\left\langle\xi_{0}^{2} \xi_{1}\right\rangle
$$

to solve (93) for $Z(\tau)$. Noting that

$$
d\left\langle\xi_{0}^{2}(t) \xi_{1}(t)\right\rangle / d t=0
$$


in equilibrium, and using (83) and (84), one can show

$$
Z(0)=-\left(1 / 2 \gamma_{1}\right)\left[\gamma_{2}\left\langle\xi_{0}{ }^{2}\right\rangle^{2}+c_{2}{ }^{\prime}\left\langle\xi_{0}{ }^{2}\right\rangle+\frac{1}{3} c_{3}\right]
$$

by evaluating

$$
2\left\langle\delta_{0}(t) \xi_{0}(t) \xi_{1}(t)\right\rangle+\left\langle{\sigma_{1}}_{1}(t) \xi_{0}^{2}(t)\right\rangle
$$

from (47) in the derivation.

Taking the Laplace transform of (89) and (93), and using

$$
\varphi(0)=\left(a_{1} /-\gamma_{1}\right)\left\langle\xi_{0}{ }^{2}\right\rangle+\left(\gamma_{2} /-\gamma_{1}\right) Z(0)
$$

[this is obtained also from (89) with $d \varphi(\tau) / d \tau=0$ at $\tau=0$ ], we obtain $\bar{\varphi}(s)$. Substitution of the latter into (81) yields the Laplace transform of the correlation function

$$
\begin{aligned}
\Phi(s)= & \epsilon\left\langle\xi_{0}^{2}\right\rangle\left[\frac{1}{s-\gamma_{1}}+\frac{a_{2}-a_{1}-2 a_{3}}{\gamma_{1}} \frac{\epsilon}{s-\gamma_{1}}\right. \\
& \left.+\frac{a_{3}-a_{2}}{\gamma_{1}} \frac{\epsilon}{s-2 \gamma_{1}}+\left(a_{2}+a_{1}\right) \frac{\epsilon}{\left(s-\gamma_{1}\right)^{2}}\right]
\end{aligned}
$$

The power spectral density follows from (97) through

$$
G(\omega)=2 \operatorname{Re}[\bar{\Phi}(i \omega)]
$$

as

$$
G(\omega)=2 \epsilon\left\langle\xi_{0}^{2}\right\rangle\left[\frac{-\gamma_{1}+2 \epsilon\left(a_{3}-a_{2}\right)}{\omega^{2}+\gamma_{1}^{2}}+\epsilon \frac{2 \gamma_{1}^{2}\left(a_{1}+a_{2}\right)}{\left(\omega^{2}+\gamma_{1}{ }^{2}\right)^{2}}+\epsilon \frac{2\left(a_{2}-a_{3}\right)}{\omega^{2}+4 \gamma_{1}^{2}}\right]
$$

where, in addition to $a_{1}$ in (90), we have defined

$$
\begin{aligned}
& a_{2} \equiv-\left(\gamma_{2} / \gamma_{1}\right)\left(\gamma_{2}+\frac{1}{2} c_{2}{ }^{\prime}\right) \\
& a_{3} \equiv-\left(\gamma_{2} / 2 \gamma_{1}\right)\left(\gamma_{2}\left\langle\xi_{0}{ }^{2}\right\rangle+c_{2}{ }^{\prime}+c_{3} / 3\left\langle\xi_{0}{ }^{2}\right\rangle\right)
\end{aligned}
$$

It is observed in (98) that a new relaxation time $1 / 2\left|\gamma_{1}\right|$ emerges when terms proportional to $\epsilon^{2}$ are included in the power spectral density. The second term may be combined with the first one within the accuracy of order $\epsilon^{2}$ as

$$
G(\omega)=2 \epsilon\left\langle\xi_{0}^{2}\right\rangle\left[\frac{-\gamma_{1}+2 \epsilon\left(a_{3}-a_{2}\right)}{\gamma_{1}{ }^{2}(1-\alpha \epsilon / 2)^{2}+\omega^{2}}+\epsilon \frac{2\left(a_{2}-a_{3}\right)}{4 \gamma_{1}{ }^{2}+\omega^{2}}\right]
$$

where

$$
\alpha=-2\left(a_{1}+a_{2}\right) / \gamma_{1}
$$

This indicates that the first relaxation time changes from $1 /\left|\gamma_{1}\right|$ to $1 /\left|\gamma_{1}\right|(1-\alpha \epsilon / 2)$ when higher order terms in $\epsilon$ are taken into account. These 
general conclusions are in complete agreement with those obtained by van Kampen ${ }^{(8)}$ in a special nonlinear model to be discussed below.

The third moment of the fluctuations vanishes in the limit of $\epsilon \rightarrow 0$ because $\xi_{0}$ was shown to be Gaussian. The first nonvanishing term in the third moment is proportional to $\sqrt{\epsilon}$, viz.,

$$
\left\langle\xi^{3}\right\rangle=\left\langle\left(\xi_{0}+\sqrt{\epsilon} \xi_{1}+\epsilon \xi_{2}\right)^{2}\right\rangle=3 \sqrt{\epsilon}\left\langle\xi_{0}^{2} \xi_{1}\right\rangle+O(\epsilon)
$$

When the system is in a stable equilibrium state, one can use (94) to calculate $\left\langle\xi_{0}^{2} \xi_{1}\right\rangle$ so that

$$
\left\langle\xi^{3}\right\rangle=2 \sqrt{\epsilon} Z(0)+O(\epsilon)
$$

where $Z(0)$ is given by $(95) .\left\langle\xi^{3}\right\rangle$ may be considered a measure of departure of the distribution of fluctuations from a Gaussian.

\section{APPLICATIONS}

As a first application of the general conclusions obtained in the previous sections, we consider Alkemade's( ${ }^{(6)}$ diode as a nonlinear model system, which was investigated in detail by van Kampen. ${ }^{(7,8)}$ The transition probability per unit time of this model is expressed in proper time units as

$$
\omega(x, r)=\delta(r,-1)+e^{-x} \delta(r, 1)
$$

where $x$ is the intensive variable, and is related to the number of excess electrons $N$ on one electrode of the diode by

$$
x=\epsilon\left[N+\frac{1}{2}\right]
$$

The smallness parameter $\epsilon$ is inversely proportional to the capacitance of the condenser. The details of this model are summarized by van Kampen ${ }^{(8)}$ and will not be reproduced here. The transition probability (105) leads to the master equation

$$
\epsilon \frac{\partial P(x, t)}{\partial t}=P(x+\epsilon)-P(x)+e^{-(x-\epsilon)} P(x-\epsilon)-e^{-x} P(x)
$$

The equilibrium distribution is calculated by van Kampen ${ }^{(8)}$ from (107) as

$$
P_{\text {eq }}(x)=P_{\text {eq }}\left(\frac{\epsilon}{2}\right) \exp \left[-\frac{1}{2 \epsilon}\left(x-\frac{\epsilon}{2}\right)^{2}\right]
$$

which is Gaussian with a mean $\epsilon / 2$.

Substituting the moments $c_{n}(x)$ obtained from (105) as

$$
c_{n}(x)=(-1)^{n}+e^{-x}
$$


into (31), (32), and (55), we obtain the evolution of the mean $\langle x\rangle=y+$ $\epsilon u_{0}+O\left(\epsilon^{3 / 2}\right)$ and the variance $\sigma=\left\langle\xi_{0}{ }^{2}\right\rangle$ as

$$
\begin{aligned}
d y / d t & =-1+e^{y} \\
d u_{0} / d t & =-u_{0} e^{-y}+\frac{1}{2} e^{-y} \sigma \\
d \sigma / d t & =-2 \sigma e^{-y}+2
\end{aligned}
$$

The equilibrium state is $y_{e}=0$, and is stable. From (111) and (112) we find $\sigma_{e}=1$ and $u_{0 e}=1 / 2$. The latter implies $\langle x\rangle_{\text {eq }}=\epsilon / 2$, which agrees with the exact value. The variance of the equilibrium is $\epsilon \sigma_{e}=\epsilon$, which also agrees with (108).

The Langevin equations for the fluctuations about the equilibrium state $y_{e}=0$ are found from (83)-(85) as

$$
\begin{aligned}
& \dot{\xi}_{0}+\xi_{0}=\varsigma_{0} \\
& \dot{\xi}_{1}+\xi_{1}=\frac{1}{2}\left(\xi_{0}{ }^{2}-1\right)+\varsigma_{1} \\
& \dot{\xi}_{2}+\xi_{2}=\frac{1}{2} \xi_{0}+\xi_{0} \xi_{1}-\frac{1}{6} \xi_{0}{ }^{3}+\delta_{2}
\end{aligned}
$$

where the properties of the random sources are given by (38)-(42), and where $\dot{\xi}_{0} \equiv d \xi_{0} / d t$.

To calculate the power spectral density, we find $\gamma_{1}=-1, \gamma_{2}=1$, $\gamma_{3}=-1, c_{2}{ }^{\prime}=-1$, and $c_{3}=0$ from (66) and (67), so that (90), (99), and (100) lead to $a_{1}=0, a_{2}=1 / 2$, and $a_{3}=0$, respectively. Substitution of these values into (101) yields

$$
G(\omega)=2 \epsilon\left[\frac{1-\epsilon}{\left(1-\frac{1}{2} \epsilon\right)^{2}+\omega^{2}}+\frac{\epsilon}{4+\omega^{2}}\right]
$$

which is identical to the result obtained by van Kampen, ${ }^{\left({ }^{(8)}\right.}$ by expanding the exact power spectral density into a power series in $\epsilon$ and retaining terms to order $\epsilon^{2}$. It is pointed out and demonstrated by van Kampen ${ }^{(7)}$ that there are terms in the exact power spectral density that cannot be obtained by an expansion method because they are not analytic in $\epsilon$. These terms are small, of infinite order in $\epsilon$, and not of practical significance.

The third moment of the distribution of $x$ is given to order $\epsilon^{1 / 2}$ by (104), which yields $\left\langle\xi^{3}\right\rangle=0$ to this order, since $Z(0)=0$. This result also is consistent with the exact equilibrium distribution (108), which is Gaussian.

A second example is the Weiss-Ising model for a ferromagnet discussed by Kubo et $a l .^{(2)} \mathrm{It}$ is characterized by

$$
\omega(x, r)=(1+x) e^{-\mu-\alpha x} \delta(r,-1)+(1-x) e^{\mu+\alpha x} \delta(r, 1)
$$

where

$$
x=\left(N_{+}-N_{-}\right) / N
$$


with $N_{+}$and $N_{-}$the numbers of plus and minus spins, and $N=N_{+}+N_{-}$. In (114), $\mu$ and $\alpha$ are proportional to the external magnetic field and molecular field, respectively. The smallness parameter $\epsilon$ is $\epsilon=2 / N$. The moments of (114) are

$$
c_{n}(x)=(-1)^{n}(1+x) e^{-\mu-\alpha x}+(1-x) e^{\mu+\alpha x}
$$

The equilibrium states of this system are obtained from $c_{1}\left(y_{e}\right)=0$ as the roots of

$$
y_{e}=\tanh \left(\mu+\alpha y_{e}\right)
$$

The interesting feature of this model is that it possesses a critical equilibrium $y_{e}=0$ when $\mu=0$ and $\alpha=1$, and a marginal equilibrium when $\alpha>1$ at a critical value of $\mu$, as can easily be verified by evaluating $c_{1}^{\prime}\left(y_{e}\right)=\gamma_{1}$, $c_{1}^{\prime \prime}\left(y_{e}\right)=\gamma_{2}$, and $c_{1}^{\prime \prime \prime}\left(y_{e}\right)=\gamma_{3}$. Since the implications of this model, such as the anomalous fluctuations, are discussed by Kubo et al., ${ }^{(2)}$ we shall not dwell on it any further.

A nuclear reactor with feedback provides another interesting nonlinear model system for the application of the general theory. It is characterized by a transition probability

$$
\begin{aligned}
\omega(x, r)= & x(\beta+\gamma x) \delta(r,-1)+\sum_{v} P_{f}(v) \alpha x \delta(r, v-1) \\
& +\sum_{m} P_{s}(m) S_{0} \delta(r, m)
\end{aligned}
$$

where $x$ is the neutron density, $\beta$ and $\alpha$ are the capture and fission rates per neutron, respectively, $\gamma$ is the feedback coefficient, $S_{0}$ is the external source per unit volume, and $P_{f}(v)$ and $P_{s}(m)$ are the numbers of neutrons per fission and per source event. This model is a generalization of the Malthus-Verhulst model in population statistics discussed by van Kampen. ${ }^{(5)}$ We shall present the implications of this model in a future work.

\section{SCALING OF CRITICAL FLUCTUATIONS}

Let $y_{e}$ be an equilibrium state for which

$$
c_{1}\left(y_{e}\right)=c_{1}{ }^{\prime}\left(y_{e}\right)=\cdots=c^{(k-1)}=0, \quad c_{1}{ }^{(k)}\left(y_{e}\right) \neq 0
$$

and assume that the intensive variable $x$ is measured from $y_{e}$. Then,

$$
c_{1}(x)=x^{k} \gamma_{k}(x)
$$

where $\gamma_{k}(x)$ is finite at $x=0$. The Langevin equation (25) becomes

$$
d x / d t=x^{k} \gamma_{k}(x)+\sqrt{\epsilon} \delta(t)
$$


where $s(t)$ satisfies [cf. (18)]

$$
\begin{aligned}
& \langle\delta(t) x(t)\rangle=\frac{1}{2} \sqrt{\epsilon}\left\langle c_{2}(x)\right\rangle \\
& \left\langle\delta(t) \delta\left(t^{\prime}\right)\right\rangle=\left\langle c_{2}(x)\right\rangle \delta\left(t-t^{\prime}\right)
\end{aligned}
$$

We assume that $c_{2}(0) \neq 0$. We want to scale $x$ and $t$ simultaneously such that we obtain a Langevin equation independent of $\epsilon$. Let

$$
x=\epsilon^{v} z, \quad t=\epsilon^{\mu} \tau
$$

Since $\delta\left(\epsilon^{\mu} \tau\right)=\epsilon^{-\mu} \delta(\tau),(122)$ leads to a scaling for the random source $\delta(t)$ as

$$
\zeta(t)=\epsilon^{-\mu / 2} q(\tau)
$$

Substitution of (124) and (123) into (120) yields

$$
d z / d \tau=z^{k} \gamma_{k}\left(\epsilon^{\nu} z\right)+q(\tau)
$$

provided that we choose

$$
\begin{aligned}
\nu & =1 /(k+1) \\
\mu & =-(k-1) /(k+1)
\end{aligned}
$$

With these choices, the scaled random source $q(\tau)$ satisfies

$$
\begin{aligned}
\langle q(\tau) z(\tau)\rangle & =\frac{1}{2}\left\langle c_{2}\left(\epsilon^{v} z\right)\right\rangle \\
\left\langle q(\tau) q\left(\tau^{\prime}\right)\right\rangle & =\left\langle c_{2}\left(\epsilon^{v} z\right)\right\rangle \delta\left(\tau-\tau^{\prime}\right)
\end{aligned}
$$

In the limit of $\epsilon \rightarrow 0$, we find the following scaled equations:

$$
\begin{aligned}
d z / d \tau & =z^{k} \gamma_{k}+q(\tau) \\
\langle q(\tau) z(\tau)\rangle & =\frac{1}{2} c_{2} \\
\left\langle q(\tau) q\left(\tau^{\prime}\right)\right\rangle & \left.=c_{2} \delta\left(\tau-\tau^{\prime}\right)\right\rangle
\end{aligned}
$$

where $\gamma_{k}=\gamma_{k}(0)$ and $c_{2}=c_{2}(0)$. For $k=3$ (critical equilibrium) we find $x=\epsilon^{1 / 4} z$ and $t=\epsilon^{-1 / 2} \tau$, and for $k=2$ (marginal equilibrium) $x=\epsilon^{1 / 3} z$ and $t=\epsilon^{-1 / 3} \tau$. Thus, the relaxation constants for the fluctuations must accumulate to zero as $\sqrt{\epsilon}$ in the critical equilibrium case, and as $\epsilon^{1 / 3}$ in the marginal equilibrium case, because $z(\tau)$ is independent of $\epsilon$ inasmuch as it satisfies (129). This is precisely the scaling rule given by Kubo et al. ${ }^{(2)}$ based on the KramersMoyal expansion of the master equation. It is interesting that the Langevin description leads to the correct scaling of the relaxation times, even though by itself it cannot yield the complete relaxation spectrum.

Multiplying (129) by $2 z(\tau)$ and using (130), we find

$$
d\left\langle z^{2}(\tau)\right\rangle / d \tau=2 \gamma_{k}\left\langle z^{k+1}\right\rangle+c_{2}
$$


which leads in equilibrium to

$$
\left\langle z^{k+1}\right\rangle=-\left(c_{2} / 2 \gamma_{k}\right)
$$

Similarly, multiplying (129) by $z(0)$ and using the causality condition $\langle q(\tau) z(0)\rangle$ for $\tau>0$, we find a similar relation for the correlation function $\phi(\tau)=\langle z(\tau) z(0)\rangle$ :

$$
d \phi(\tau) / d \tau=\gamma_{k}\left\langle z^{k}(\tau) z(0)\right\rangle
$$

Suppose $z(\tau)$ is Gaussian (for $k=1$ this is an exact property). Then, for $k=3$ we can obtain an equation for the variance $\sigma(\tau)=\left\langle z^{2}\right\rangle$ from (132) using $\left\langle z^{4}\right\rangle=3\left\langle z^{2}\right\rangle^{2}$,

$$
d \sigma(\tau) / d \tau=6 \gamma_{3} \sigma^{2}(\tau)+c_{2}
$$

Using $\left\langle z^{3}(\tau) z(0)\right\rangle=3 \phi(\tau) \phi(0)$ for a Gaussian process [cf. (87)] in (134), we find

$$
d \phi(\tau) / d \tau=3 \gamma_{3} \phi(\tau) \phi(0)
$$

We assume $\gamma_{3}<0$, so that $\sigma(\tau)$ in (135) relaxes to an equilibrium

$$
\sigma_{e}=\left(c_{2} / 6\left|\gamma_{3}\right|\right)^{1 / 2}
$$

If $\sigma_{0}$ is the initial value at $t=0$, the solution of (135) can be shown to be, in real time,

$$
\sigma(t)=\sigma_{e}\left(1+K e^{-\lambda t}\right) /\left(1-K e^{-\lambda t}\right)
$$

where

$$
\begin{aligned}
& K=\left(\sigma_{0}-\sigma_{e}\right) /\left(\sigma_{0}+\sigma_{e}\right) \\
& \lambda=2 \sqrt{\epsilon}\left(6 c_{2}\left|\gamma_{3}\right|\right)^{1 / 2}
\end{aligned}
$$

Near the equilibrium $\sigma(t)$ behaves as

$$
\sigma(t) \approx \sigma_{e}\left(1+2 K e^{-\lambda t}\right)
$$

The relaxation of the correlation function in equilibrium is determined from (136) as

$$
\phi(t)=\sigma_{e} e^{-\lambda t / 4}
$$

The relaxation frequency $\lambda$ in (140) approaches zero as $\sqrt{\epsilon}$ in the limit of $\epsilon \rightarrow 0$, as pointed out earlier. The expression (140) is in qualitative agreement with Eq. (187) of Kubo et al. ${ }^{(2)}$ for the asymptotic evaluation of relaxation frequencies. The dependence of $\lambda$ on $\left|\gamma_{3}\right|, c_{2}$, and $\epsilon$ is identical in both results, which differ from each other only by a numerical factor. However, this agreement may be fortuitous on our part because we do not have a real justification for assuming the distribution of $z$ to be Gaussian when $k>1$. 
The mean of $z$ when $k=3$ follows from (129) as $\langle\dot{z}\rangle=\gamma_{3}\left\langle z^{3}\right\rangle$, which indicates that the Gaussian assumption for which $\left\langle z^{3}\right\rangle=0$ is consistent with the equilibrium condition $\langle\dot{z}\rangle=0$. In the case of $k=2$ corresponding to a marginal equilibrium, the Gaussian assumption implies through (132) that $\left\langle z^{2}\right\rangle \sim c_{2} \tau$, i.e., the distribution broadens in time without reaching an equilibrium.

\section{CONCLUSIONS}

In this paper we have investigated the foundation of the Langevin equation method of studying fluctuations in a nonlinear Markov process, and demonstrated how successive Langevin equations can be obtained by expanding the mean, fluctuations, and random source into a series in powers of $\epsilon$. To the lowest order in $\epsilon$ the Langevin equation is linear with a timevarying coefficient $\left[c_{1}{ }^{\prime}(y)\right.$ in Eq. (53)] and contains a Gaussian white noise source. The power spectral density of this source is also time dependent [ $c_{2}(y)$ in Eq. (38d)] in general. The implicit time dependence of $c_{1}{ }^{\prime}(y, t)$ and $c_{2}(y, t)$ through $y(t)$ is given by the deterministic equation $\dot{y}=c_{1}(y, t)$. In time-invariant systems they do not depend on time explicitly. Thus, the deterministic equation and the Langevin equation provide a general description of the fluctuation even in nonstationary systems, provided $c_{2}(y, t)$ is known.

The description of the fluctuations about equilibrium requires $c_{2}\left(y_{e}\right)$ in addition to $c_{1}(y)$, which can be obtained from the macroscopic rate equation of the system. ${ }^{5}$ Since $c_{2}\left(y_{e}\right)=2\left|c_{1}{ }^{\prime}\left(y_{e}\right)\right| \sigma_{e}$, where $\sigma_{e}$ is the equilibrium value of the variance, a knowledge of $\sigma_{e}$ either from measurement or from thermodynamic arguments, if the system is in thermal equilibrium, completes the description of the fluctuations to the lowest order in $\epsilon$.

The calculation of the power spectral density in equilibrium correct to order $\epsilon^{2}$ [cf. Eq. (98) or (101)] requires $c_{1}^{\prime \prime}\left(y_{e}\right), c_{1}^{\prime \prime}\left(y_{e}\right), c_{2}{ }^{\prime}\left(y_{e}\right)$, and $c_{3}\left(y_{e}\right)$ in addition to $c_{1}{ }^{\prime}\left(y_{e}\right)$ and $c_{2}\left(y_{e}\right)$. Of course, they are easily calculated if the transition probability $w(x, r)$ is known. However, the main advantage of the Langevin equation approach is that it enables one to determine the power spectral density in terms only of the parameters appearing in the macroscopic rate equation and the variance, without requiring any other statistical knowledge. We find that in nonlinear systems this is not possible, as one would expect, if terms of higher order in $\epsilon$ than the first are included in the power

${ }^{5}$ It is not clear whether the macroscopic rate equation of the system can be interpreted as the deterministic equation $\dot{y}=c_{1}(y)$, which is the bare transport equation of the system in conventional terminology. ${ }^{(13)}$ One may argue that the macroscopic equation, which is often known only phenomenologically, is already fluctuation-renormalized, ${ }^{(13)}$ so that this identification can only be an approximation. 
spectral density. However, the additional parameters $c_{2}{ }^{\prime}\left(y_{e}\right)$ and $c_{3}\left(y_{e}\right)$ may be obtained experimentally by measuring the variance as a function of the equilibrium $y_{e}$ [if it is possible to vary $y_{e}$ by changing a parameter in $c_{1}\left(y_{e}\right)$ without changing $\left.c_{2}(y)\right]$ and the third moment of the fluctuations. The latter yields $c_{3}\left(y_{e}\right)$ through (104) if $c_{2}{ }^{\prime}\left(y_{e}\right)$ is already determined.

If the transition probability $w(x, r)$ is known, as is the case in the physical models presented in Section 7, one can of course attempt to solve the master equation directly as was done by van Kampen ${ }^{(1)}$ and Kubo et al. ${ }^{(2)}$ The Langevin equation method in such cases may be considered as an alternative approach to the study of fluctuations in nonlinear systems.

\section{APPENDIX. THE PROOF OF (80a)}

Assume $t>t^{\prime}$ and multiply (83) by $\xi_{1}\left(t^{\prime}\right)$, multiply (84) by $\xi_{0}\left(t^{\prime}\right)$, average, and add the resulting equations. Using (42a), one finds

$$
\partial Q\left(t, t^{\prime}\right) / \partial t=\gamma_{1} Q+\frac{1}{2} \gamma_{2}\left\langle\xi_{0}^{2}(t) \xi_{0}\left(t^{\prime}\right)\right\rangle, \quad t>t^{\prime}
$$

where

$$
Q\left(t, t^{\prime}\right) \equiv\left\langle\xi_{0}(t) \xi_{1}\left(t^{\prime}\right)\right\rangle+\left\langle\xi_{0}\left(t^{\prime}\right) \xi_{1}(t)\right\rangle
$$

Since $\xi_{0}(t)$ is Gaussian, the last term on the right-hand side is zero, so that we have

$$
Q\left(t, t^{\prime}\right)=\left\{\exp \left[\gamma_{1}\left(t-t^{\prime}\right)\right]\right\} Q\left(t^{\prime}, t^{\prime}\right), \quad t>t^{\prime}
$$

Now multiply (83) by $\xi_{1}(t),(84)$ by $\xi_{0}(t)$, and again use (42a) to get

$$
d Q(t, t) / d t=2 \gamma_{1} Q(t, t)
$$

In equilibrium $\dot{Q}(t, t)=0$, so that $Q(t, t)$ and hence $Q\left(t, t^{\prime}\right)$ vanish for all $t$, proving (80a).

\section{ACKNOWLEDGMENTS}

The author expresses his gratitude to Mr. Mustafa Benmouna for carefully reading the manuscript and reproducing all the results of this paper, and also to Mr. Shikoh Itoh, Nuclear Engineering Department, Nagoya University, Japan, for his critical remarks on Section 3.

\section{REFERENCES}

1. N. G. van Kampen, Can. J. Phys. 39:551 (1961).

2. R. Kubo, K. Matsuo, and K. Kitahara, J. Stat. Phys. 9:51 (1973).

3. H. Mori, Prog. Theor. Phys. $52: 433$ (1974).

4. H. Haken, Rev. Mod. Phys. 17:67 (1975); J. Keizer, J. Chem. Phys. 63:398 (1975); $63: 5037$ (1975); 64: 1679 (1976). 
5. N. G. van Kampen, in Irreversibility in the Many-Body Problem, J. Biel and J. Rae, eds., Plenum Press, New York (1972), pp. 369-386.

6. C. T. J. Alkemade, Physica 24:1029 (1958).

7. N. G. van Kampen, J. Math. Phys. 2:592 (1961).

8. N. G. van Kampen, Fluctuations in Solids, R. E. Burgess, ed., Academic, New York (1965); Physica 26:585 (1960).

9. M. Lax, Rev. Mod. Phys. 32:25 (1960).

10. L. Arnold, Stochastic Differential Equations: Theory and Applications, Wiley, New York (1973), Chapter 10.

11. M. J. Lighthill, An Introduction to Fourier Analysis and Generalized Functions, Cambridge Univ. Press, London (1959).

12. M. Lax, Rev. Mod. Phys. 38:541 (1966).

13. K. Sture, J. Nordholm, and Robert Zwanzig, J. Stat. Phys. 11:2 (1974). 